\title{
Enfermedad de Moyamoya
}

\author{
Moyamoya disease
}

\author{
Adolfo Enrique Castro-Beltrán, María Cecilia Diaz, Carlos Donado-Canedo, \\ Nehomar Pájaro-Galvis, Loida Camargo-Camargo • Cartagena (Colombia)
}

DOI: https://doi.org/10.36104/amc.2020.1424

\section{Resumen}

La enfermedad de Moyamoya es una enfermedad neurovascular crónica caracterizada por una estenosis progresiva bilateral de las arterias carótidas internas, con la formación anormal de nuevos vasos sanguíneos, cuyo bloqueo en flujo sanguíneo ocasiona variadas manifestaciones clínicas y complicaciones asociadas a eventos cerebrovasculares (isquémicos o hemorrágicos) agudos que pueden llegar a ser incluso mortales. El presente artículo pretende realizar una aproximación acerca de esta entidad, su incidencia, prevalencia, formas de presentación y medidas terapéuticas y pronostico mediante el reporte de un caso clínico. (Acta Med Colomb 2020; 45. DOI: https:// doi.org/10.36104/amc.2020.1424).

Palabras clave: enfermedad de Moyamoya, trastornos cerebrovasculares, enfermedad cerebrovascular gangliobasal.

\section{Abstract}

Moyamoya disease is a chronic neurovascular disease characterized by progressive bilateral stenosis of the internal carotid arteries with abnormal formation of new abnormal blood vessels whose blockage of blood flow causes a variety of clincal manifestations and complications associated with acute cerebrovascular events (ischemic or hemorrhagic), which may even be fatal. This article seeks to describe this entity, its incidence, prevalence, forms of presentation, therapeutic measures and prognosis through a clinical case report. (Acta Med Colomb 2020; 45. DOI: https:// doi.org/10.36104/amc.2020.1424).

Key words: moyamoya disease, cerebrovascular disorders, basal ganglia cerebrovascular disease.
Dres. Adolfo Enrique Castro Beltrán y María Cecilia Díaz: Residentes de Neurología Clínica, Universidad del Sinú, Seccional Cartagena; Dr. Carlos Donado-Canedo: Médico General, Unidad de Cuidados Intensivos Nuevo Hospital Bocagrande; Dr. Nehomar Pájaro-Galvis: Residente de Medicina Interna, Universidad del Sinú Seccional Cartagena; Dra. Loida Camargo-Camargo: Docente de Postgrado de Neurología, Universidad del Sinú Seccional Cartagena. Cartagena (Colombia). Correspondencia: Dr. Adolfo Enrique Castro Beltrán. Cartagena (Colombia)

E-mail: adolfohdyes1@gmail.com Recibido: 16/VII/2019 Aceptado: 06/II/2020

\section{Introducción}

La enfermedad de Moyamoya es una enfermedad crónica que se caracterizada por estenosis progresiva bilateral de la porción terminal de la arteria carótida interna y sus ramas proximales con la consiguiente neoformación de una red arterial colateral anormal de las arterias que forman el polígono de Willis con manifestaciones clínicas variables y nada específicas (1-3).

Esta es una enfermedad rara con muy baja prevalencia entre hispanos siendo las convulsiones en combinación con los síntomas atribuidos a accidentes cerebrovasculares de las manifestaciones menos frecuentes en adultos $(9,21)$.

El objetivo de este artículo es presentar el caso clínico de una paciente con este trastorno que llegó con una crisis tonicoclónico generalizada precedida de cefalea con posterior recuperación neurológica total a la cual se le documentó hemorragia gangliobasal derecha y arteriopatía bilateral típi- ca de esta enfermedad mediante angiografía por sustracción digital cerebral $(3,5)$.

\section{Reporte de caso}

Paciente femenina de 45 años de edad de etnia mestiza sin antecedentes patológicos, quien ingresa al servicio de urgencias de un hospital de cuarto nivel de la ciudad de Cartagena (Bolívar) el día $1^{\circ}$ de julio de 2019 con cuadro clínico de una semana de evolución consistente en cefalea holocraneana, de moderada intensidad, con posterior aparición de un episodio convulsivo tonicoclónico generalizado, con relajación de esfínteres, sin especificarse la duración de la crisis. Al ingreso al servicio de urgencias tensión arterial de 140/90 y restantes signos vitales normales sin déficit neurológico focal. Se realizó una tomografía de cráneo simple evidenciando foco hemorrágico intraparenquimatoso de localización gangliobasal derecho, leve enfermedad multifocal 
isquémica de pequeños vasos, foco malásico occipital derecho y evento isquémico antiguo frontal izquierdo adyacente al ventrículo lateral izquierdo (Figura 1). En los paraclínicos iniciales no se encontró alteración en la analítica sanguínea con reporte de hemoleucograma y recuento de plaquetas normales, azoados, ionograma y tiempos de coagulación dentro de límites de normalidad. Se realizó panangiografía cerebral encontrando como hallazgos: adelgazamiento de arterias carótidas internas y de cerebrales posteriores (observable esto último en el $50 \%$ de los casos) con presencia de neovascularización anormal a nivel de núcleos de la base, compatible con enfermedad de Moyamoya (Figura 2). Con estos resultados se evaluó en junta médica de neurocirugía la conducta a seguir, considerando que se puede beneficiar de manejo endovascular; sin embargo, ante el alto riesgo de recurrencia del sangrado, se postergó la realización del mismo al menos cuatro semanas.

\section{Discusión}

La enfermedad de Moyamoya es una enfermedad crónica que se caracterizada por estenosis progresiva bilateral de la porción terminal de la arteria carótida interna y sus ramas proximales con la consiguiente neoformación de una red arterial colateral anormal de las arterias que forman el polígono de Willis de etiología hasta ahora desconocida (1-3). El término proviene de la apariencia similar al "humo de cigarro en el aire" (Moyamoya en japonés) de las arterias colaterales observadas por angiografía que se forman de forma progresiva durante la evolución de la enfermedad (2).

El caso que presentamos hace referencia a esta enfermedad, la cual hay que diferenciar del síndrome de Moyamoya que está ligado a lesiones vasculares unilaterales asociadas con otras entidades como neurofibromatosis, anemia de

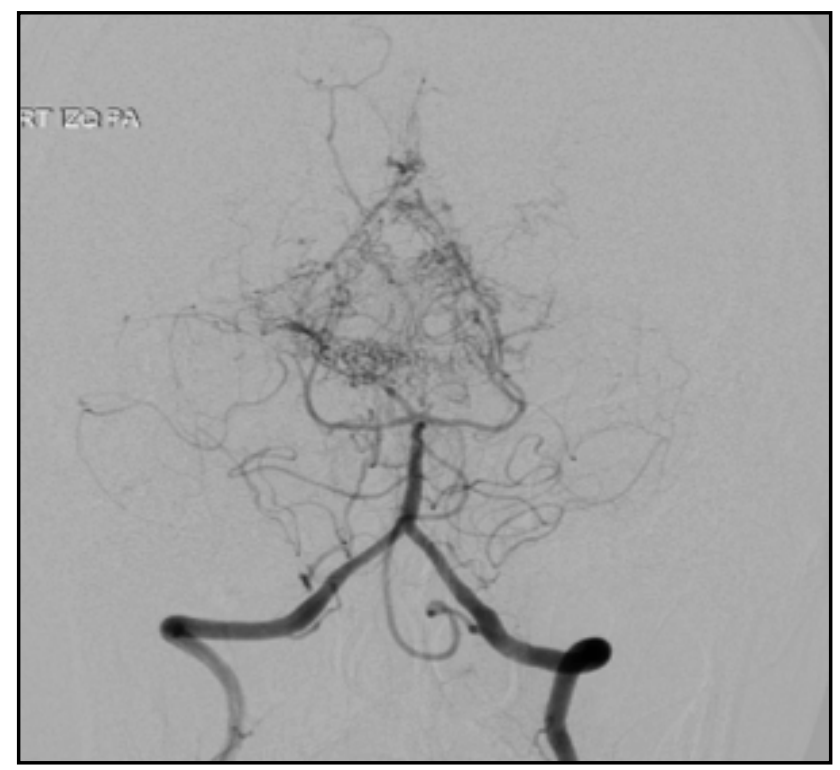

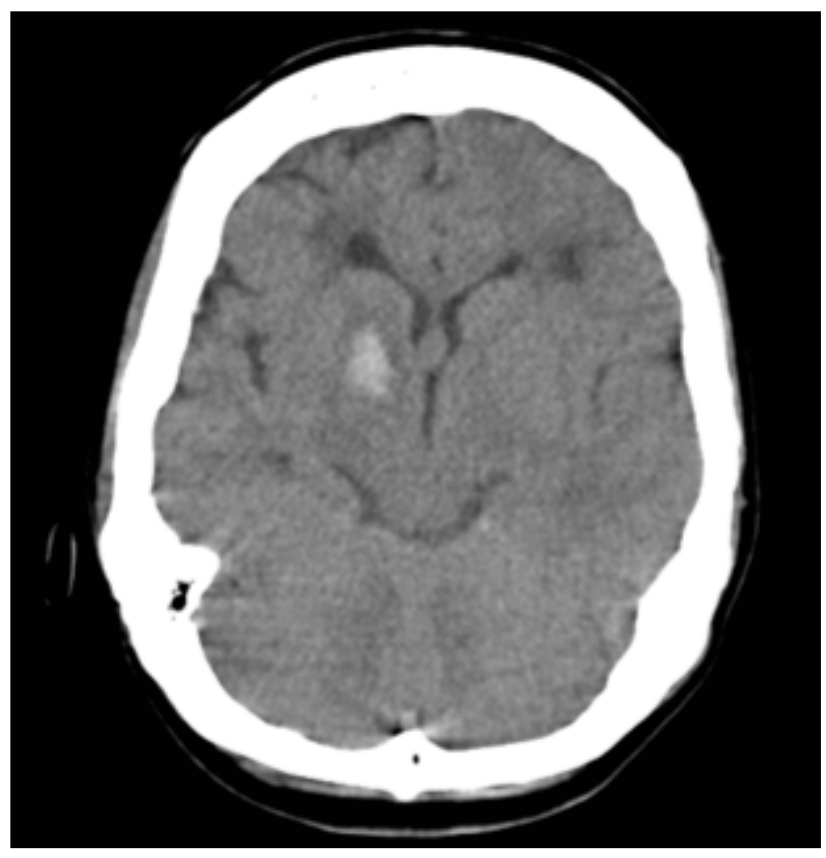

Figura 1.TC de cráneo sin contraste. Corte axial que evidencia hipodensidad nucleobasal derecha sugestivo de sangrado.

células falciformes, síndrome de Down, meningitis, trauma, enfermedades autoinmunes, secundarias a radiación craneal y enfermedad de Graves $(4,6)$.

Esta entidad suele ser más frecuente en individuos de origen asiático, y menos en personas de origen hispano, como se ha demostrado en algunos estudios. Sin embargo, puede afectar a personas de cualquier etnia. En Japón se ha encontrado una prevalencia de 3.16 casos por 100000 habitantes, mientras que en Estados Unidos se ha encontrado

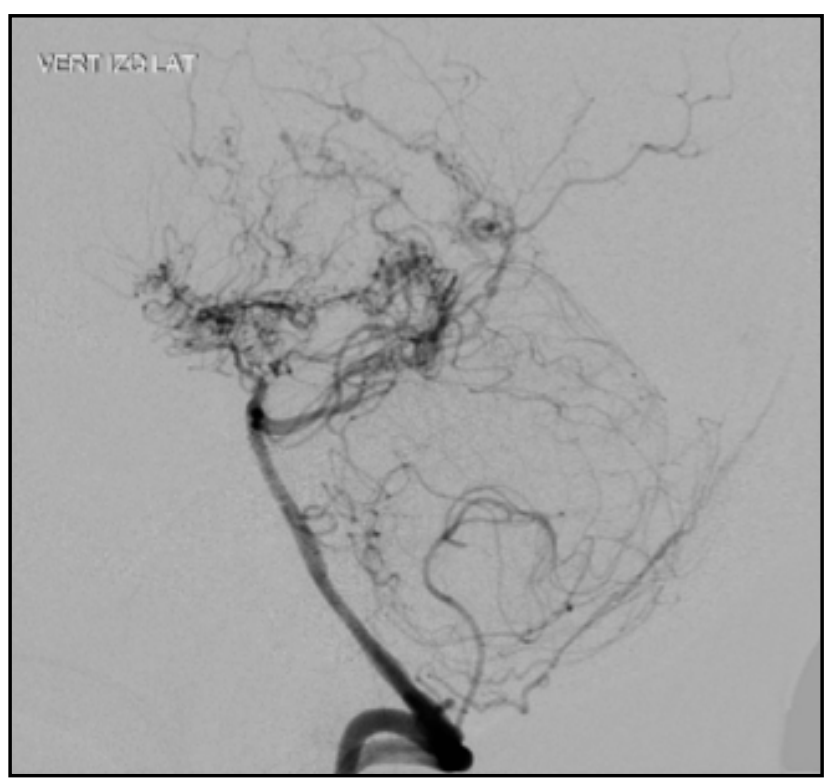

Figura 2. Arteriografía de sustracción digital cerebral, en donde se observa en el plano frontal y lateral, estenosis bilateral de la a. cerebral posterior con desarrollo de colaterales anormales que en secuencia simularían una "bocanada de humo". 
que la prevalencia de la enfermedad es de 0.086 por cada 100 000 habitantes. Afecta más a mujeres que a hombres en una proporción de 2:1, y se han reportado dos picos de incidencia: niños a los 5-9 años y adultos entre 40-50 años (más común) (7-9). En Colombia para el 2013 sólo se habían descrito cuatro casos de enfermedad de Moyamoya y todos correspondían a niños (22), mientras en 2015 se reportó solo un caso de un paciente adulto femenino con desenlace fatal (23).

Las manifestaciones clínicas de esta enfermedad varían con la edad, encontrándose que los infantes de 5-9 años con enfermedad de Moyamoya típicamente se presentan con ictus arteriales isquémicos o accidentes isquémicos transitorios (AIT) (11), mientras que en los adultos de 40-49 años se han observado variaciones geográficas, siendo más frecuentes las hemorragias intracraneales en la población asiática en comparación con la estadounidense $(1,10)$. Como se observó, nuestra paciente tenía los factores sociodemográficos de edad, etnia mestiza, sexo femenino y además el proceso hemorrágico característico de la edad adulta en esta enfermedad.

La presentación típica en adultos incluye: convulsiones, accidente cerebrovascular isquémico, hemorrágico o transitorio (signo-sintomatología focal dependiendo de sitio de lesión), deterioro cognitivo, cambio de personalidad y cefalea. Las variaciones individuales en el grado de compromiso arterial, la progresión de la estenosis, las regiones isquémicas de la corteza y la respuesta a la reducción del suministro de sangre ayudan a entender la amplia gama de presentaciones clínicas.

En nuestro caso la enfermedad de Moyamoya se presentó como un primer episodio convulsivo generalizado con posterior recuperación, sin un aparente déficit motor o cognitivo derivado de una hemorragia intraparenquimatosa nucleobasal derecha detectada por tomografía de cráneo simple, hallazgo que corresponde con la literatura, la cual menciona que la hemorragia intraparenquimales más común que sus formas intraventricular o subaracnoidea en pacientes con esta enfermedad y que las localizaciones más frecuentes son los núcleos de la base, el lóbulo temporal medial y el tálamo (1, 4). Entre las posibles causas de la hemorragia en pacientes con enfermedad de Moyamoya destacan la ruptura de alguna de las colaterales frágiles y/o de algún aneurisma formado por las alteraciones en el flujo cerebral (zonas de altas y bajas presiones que se forman durante la enfermedad). Los sitios más comunes de formación de estos aneurismas son en la parte terminal de la arteria basilar y en la arteria comunicante posterior. La ubicación de la hemorragia en nuestro caso planteó el diagnóstico diferencial con la ruptura aneurismática y la asociada a una malformación arteriovenosa.

Para el diagnóstico se utilizan diversas pruebas que evalúan la vasculatura cerebral como la angiotomografía y la angiorresonancia con buenos resultados. Sin embargo, la panangiografía cerebral (también conocida como angiografía por sustracción digital) es el "gold standard" en el diagnóstico definitivo de la enfermedad de Moyamoya al observarse el aspecto arteriográfico característico. Ésta detecta la estenosis de la porción terminal de la arteria carótida interna y sus ramas proximales así como también la formación de la red de colaterales (4). La panangiografía cerebral en nuestro caso fue solicitada como parte del estudio de hemorragia intracraneal en un paciente joven y evidenció adelgazamiento progresivo de ambas arterias carótidas internas con presencia de neovascularización y desaparición bilateral de arterias cerebrales media y anterior.

No existe una terapéutica capaz de detener la progresión o revertir los cambios arteriales que se producen en los pacientes con enfermedad de Moyamoya. El tratamiento está dirigido a aliviar la sintomatología y a prevenir la recurrencia de accidentes cerebrovasculares y limitar el deterioro cognitivo (12). Existen medidas médicas y quirúrgicas. Entre las medidas médicas se recomienda el uso de agentes antiplaquetarios excepto en aquellos pacientes que se presentan con una hemorragia intracraneal. También se deben evitar periodos de deshidratación, la hiperventilación, y en aquellos pacientes cuya presentación sea con migraña se deben usar fármacos como el topiramato y no se recomienda el uso de antiinflamatorios no esteroideos (13). La medida quirúrgica más importante en estos pacientes es la revascularización (ya sea directa, indirecta o combinada) cuyo objetivo general es mejorar el flujo sanguíneo cerebral (14). Diversos estudios han mostrado que la revascularización en los pacientes con enfermedad de Moyamoya produce una disminución en la tasa de recurrencia de accidentes cerebrovasculares (isquémicos y hemorrágicos), reducción de la sintomatología (como la cefalea), mejoría del estado clínico funcional e incluso una mejoría de las alteraciones cognitivas, con un adecuado perfil de seguridad (15-18), motivo por el cual es una medida recomendada por la Academia Americana del Corazón (AHA por sus siglas en inglés) (19). Además, Liu et al, reportaron que la terapia de revascularización tiene un impacto positivo en la prevención de resangrado en aquellos pacientes con presentación hemorrágica (20). Nuestro caso tiene indicación de terapia de revascularización (avalada en junta médica), la cual se difirió a un mes por el sangrado intracerebral reciente.

La enfermedad de Moyamoya se debe sospechar en niños o adultos menores de 50 años, con manifestaciones clínicas de enfermedad cerebrovascular isquémica y/o hemorrágica aguda sin factores de riesgo, con o sin historia previa de episodios de déficit o focalización neurológica. Es importante establecer de forma rápida el diagnóstico, debido a que la evidencia nos demuestra que las medidas terapéuticas, tanto médicas como quirúrgicas se asocian a un mejor pronóstico siendo este último el de elección y de mejores resultados. El pronóstico de los pacientes con esta enfermedad no es totalmente predecible.

\section{Referencias}

1. Scott RM, Smith ER. Moyamoya disease and moyamoya syndrome. $N$ EnglJ Med. 2009; 360(12):1226-37.

2. Suzuki J, Takaku A. Cerebrovascular moyamoya disease. Disease showing abnormal net-like vessels in base of brain. Arch Neurol. 1969;20(3):288-99. 
3. Research Committee on the Pathology and Treatment of Spontaneous Occlusion of the Circle of Willis, Health Labour Sciences Research Grant for Research on Measures for Infractable Diseases. Guidelines for diagnosis and treatment of moyamoya disease (spontaneous occlusion of the circle of Willis). Neurol Med Chir (Tokyo). 2012; 52(5): 245-66.

4. Smith ER, Scott RM. Moyamoya: epidemiology, presentation, and diagnosis. Neurosurg Clin N Am. 2010;21(3):543-51.

5. Ramírez-Quiñones JA, Barrientos-Imán DM, Calle-La RP, Ecos-Quispe RL, Novoa-Mosquera ME, Valencia-Chávez AM, et al. Enfermedad de Moyamoya: reporte de un caso. Rev Neuropsiquiatr. 2015;78(3):165-170

6. Lizarazo J, Niño F, Alvarado H, Castro N. Síndrome moyamoya y enfermedad de Graves en una mujer joven. Acta Med Colom. 2013;38(4): 262-267

7. Baba T, HoukinK, Kuroda S. Novel epidemiological features of moyamoya disease. J Neurol Neurosurg Psychiatry. 2008;79(8):900-4

8. Ahn IM, Park DH, Hann HJ, Kim KH, Kim HJ, Ahn HS. Incidence, prevalence, and survival of moyamoya disease in Korea: a nationwide, population based study. Stroke. 2014; 45(4):1090-5.

9. Uchino K, Johnston SC, Becker KJ, Tirschwell DL. Moyamoya disease in Washington State and California. Neurology. 2005;65(6):956-8.

10. Hallemeier CL, Rich KM, Grubb RL Jr, Chicoine MR, Moran CJ, Cross DT 3rd, Zipfel GJ, Dacey RG Jr, Derdeyn CP. Clinical features and outcome in North American adults with moyamoya phenomenon. Stroke. 2006 Jun;37(6):1490-6.

11. Fung LW1, Thompson D, Ganesan V. Revascularisation surgery for paediatric moyamoya: a review of the literature. Childs Nerv Syst. 2005 May;21(5):358-64.

12. Kronenburg A, Braun KPJ, vander Zwan A, Klijn CJM. Recent advances in moyamoya disease: pathophysiology and treatment. Curr Neurol Neurosci Rep. 2014;14(1):423.

13. Smith ER. Moyamoya arteriopathy. Curr Treat Options Neurol. 2012;14(6):549-56.
14. Pandey P, Steinberg GK. Neurosurgical advances in the treatment of moyamoya disease. Stroke. 2011; 42(11): 3304-10.

15. Guzman R, Lee M, Achrol A, Bell-Stephens T, Kelly M, Do HM, et al. Clinical outcome after 450 revascularization procedures for moyamoya disease. J Neurosurg. 2009;111(5):927-35.

16. Lee JY, Phi JH, Wang KC, Cho BK, Shin MS, Kim SK. Neurocognitive profiles of children with moyamoya disease before and after surgical intervention. Cerebrovasc Dis. 2011; 31(3): 230-7.

17. Bao XY, Duan L, Li DS, Yang WZ, Sun WJ, Zhang ZS, et al. Clinical features, surgical treatment and long-term outcome in adult patients with Moyamoya disease in China. Cerebrovasc Dis. 2012;34(4):305-13.

18. Duan L, Bao XY, Yang WZ, Shi WC, Li DS, Zhang ZS, et al. Moyamoya disease in China: its clinical features and outcomes. Stroke. 2012; 43(1): 56-60.

19. Roach ES, Golomb MR, Adams R, Biller J, Daniels S, deVeber G, et al. Management of stroke in infants and children a scientific statement from a special writing Group of the American Heart Association Stroke Council and the Council on Cardiovascular Disease in the Young. Stroke. 2008; 39(9): 2644-91.

20. Liu X, Zhang D, Wang S, Zhao Y, Teo M, Wang R, et al. Clinical features and long-term outcomes of moyamoya disease: a single-center experience with 528 cases in China. J Neurosurg. 2015;122(2): 392-9.

21. Kim JS. Moyamoya disease: epidemiology, clinical features, and diagnosis. $J$ Stroke.18(1): 2-11, 2016

22. Eugenia Espinosa, Angela Ortiz, Sandra Ardila, Lisseth Cabarcas, Nury Mancilla. Síndrome y enfermedad de Moyamoya: reporte de 4 casos pediátricos. Acta Neurol Colomb 2011;27:165-171.

23. Ledmar Vargas, Sergio Sánchez, Álvaro Suarez, Oscar Jiménez. Enfermedad de moyamoya: a propósito de un caso. Acta Neurol Colomb. 2016; 32(4): 310-313. 